\title{
Estimation of scattering from a moist rough surface with spheroidal dust particles
}

\author{
MUKESH KUMAR \\ B.R.A. Bihar University, Muzaffarpur 842 001, India
}

MS received 13 October 2007

\begin{abstract}
The scattering from moisture rough surface with spheroidal dust particles having surface with spheroidal dust particles has recently received much attention. In part due to the recent prediction and observation of the spheroidal dust particles in rough surfaces under elastic wave by the Kirchhoff scattering model and scalar approximation with slope. Our analysis shows that the scattering depends on the moisture (2-4.5\%) with spheroidal dust particles. At slightly moisture rough surface the dielectric properties increase with change in field amplitude in a rough surface with spheroidal dust particles.
\end{abstract}

Keywords. Surface permittivity; backscattering; surface roughness; dust particles.

\section{Introduction}

Scattering from a rough surface has been an active area of study. The basic assumption of the Kirchhoff method deals with the plane boundary reflection condition at every point on the surface. Thus, when statistical surfaces are considered then the roughness of the surfaces are considered for its main parameters. It has been found that the correction length $(\ell)$ must be larger than the electromagnetic wavelength. This implies that the surface permittivity has good agreement with scattering models and with multiple scattering and shadowing. The backscattering curve drops off more slowly with increasing incident angle at the rough surface, if the surface standard deviation is to be less than $5.5 \%$ of the magnetic wavelength. We emphasize that the surface deviation is responsible for scattering at a given electromagnetic wavelength.

\section{Theoretical consideration (basic theory)}

We consider a horizontally rough surface with slight percentage of moisture $(2-4.5 \%)$ with spheroidal dust particles.

We consider that the interface is confined with random function, $Z(x, y)$. The random function may be expressed under this condition as follows

$$
Z(x, y)=\frac{1}{2 \pi} \int_{-\infty}^{+\infty} \int_{-\infty}\left(K_{x}, K_{y}\right) \exp \left(j K_{x} x+J K_{y} \cdot y\right) \mathrm{d} K_{x} \cdot \mathrm{d} K_{y} .
$$

(mukbihar@rediffmail.com)
Also,

$$
Z\left(K_{x}, K_{y}\right)=\frac{1}{2 \pi} \int_{-\infty}^{+\infty} \int_{-\infty} Z(x, y) \exp \left(-j K_{x} x-j K_{y} \cdot y\right) \mathrm{d} x \cdot \mathrm{d} y .
$$

Now, the incident scattered field is supposed to be horizontally polarized elastic wave propagating through the $x, z$ plane, then the total scattered fields due to moisture spheroidal dust particles in rough surface is given as (Dobson and Ulaby 1981),

$$
\begin{aligned}
E_{x} & =\frac{1}{2 \pi} \int^{\infty} \int_{-\infty} U_{x}\left(K_{x}, K_{y}\right) f \mathrm{~d} K_{x} \cdot \mathrm{d} K_{y}, \\
E_{y} & =\frac{1}{2 \pi} \int^{\infty} \int_{-\infty} U_{y}\left(K_{x}, K_{y}\right) f \mathrm{~d} K_{x} \cdot \mathrm{d} K_{y} \\
& +e^{-j k \sin \theta}\left(e^{-j k_{z} \cos \theta}+R e^{-j k_{z} \cos \theta}\right), \\
E_{z} & =\frac{1}{2 \pi} \int_{-\infty}^{\infty} \int_{z}\left(K_{x}, K_{y}\right) f \mathrm{~d} K_{x} \cdot \mathrm{d} K_{y},
\end{aligned}
$$

where $f=\exp \left(j K_{x} x+j K_{y} y-j K_{z} z\right), \quad K_{z}=\left(K^{2}-K_{x}^{2}-K_{y}^{2}\right)^{1 / 2}$, $K$ the wave number in rough surface with spheroidal dust

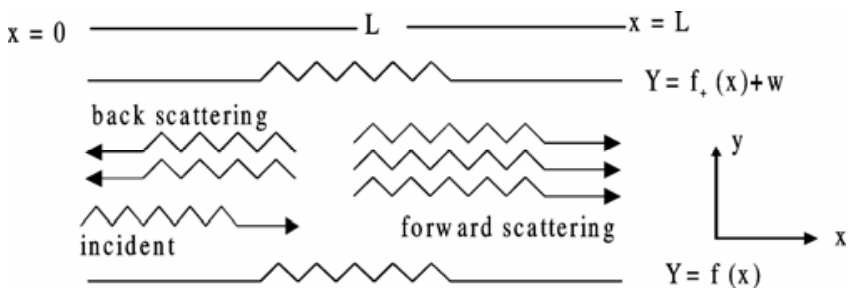

Figure 1. 2D model used for calculation of the scattering of elastic wave by rough surface. 
particles, $\mu_{\mathrm{r}}$ the permeability of the moisture rough surface w.r.t. elastic surface, $\varepsilon_{\mathrm{r}}$ the permittivity of the moisture rough surface w.r.t. elastic surface and also $K^{1}=2\left(\mu_{\mathrm{r}} \varepsilon_{\mathrm{r}}\right)^{1 / 2}, K_{2}^{1}=\left(K_{1}^{2}-K_{x}^{2}-K_{y}^{2}\right)^{1 / 2}$.

Now, the total scattered field with moisture spheroidal particles may be given as (Fawwaz et al 1981),

$$
\begin{aligned}
& E_{x}^{1}=\frac{1}{2 \pi} \int^{\infty} \int_{-\infty} D_{x}\left(K_{x} \cdot K_{y}\right) g \mathrm{~d} K_{x}-\mathrm{d} K_{y}, \\
& E_{y}^{1}=\frac{1}{2 \pi} \int^{\infty} \int_{-\infty} D_{y}\left(K_{x} \cdot K_{y}\right) g \mathrm{~d} K_{x} \mathrm{~d} K_{y}, \\
& E_{z}^{1}=\frac{1}{2 \pi} \int_{-\infty}^{\infty} \int_{z}\left(K_{x} \cdot K_{y}\right) g \mathrm{~d} K_{x} \mathrm{~d} K_{y},
\end{aligned}
$$

where

$$
g=\exp \left(j K_{x}^{x}+j K_{y} y+j K_{z}^{1} \cdot Z\right) .
$$

Now the expression for transmission loss and absorption loss for the wave guide scattering pattern model under rough surface can be written as (Shimaru 1978),

$$
\begin{aligned}
& P_{\mathrm{abs}}=|T|=1+|R|=\frac{2 \mu_{\mathrm{r}} K \cos \theta}{\mu_{\mathrm{r}} \cdot K \cos \theta+K^{\prime} \cos \theta}, \\
& P_{\mathrm{abs}}=\frac{2 \mu_{\mathrm{r}} \cos \theta}{\mu_{\mathrm{r}} \cos \theta+\left(\mu_{\mathrm{r}} \varepsilon_{\mathrm{r}}-\sin ^{2} \theta\right)^{1 / 2}} .
\end{aligned}
$$

\subsection{Mode scattering}

As discussed in the first-section, we used a scalar model for the elastic waves. We consider a (2D) wave guide like structure extended in the $x$-direction and bounded at $y=0$ and $W$ in the presence of rough surface. We assume Neumann boundary conditions at the edges of the wave guide corresponding to a stress free boundary condition for the elastic waves. The boundaries do not support the models with zero cutoff frequency. We calculate the scattering process by considering an elastic wave propagation in the wave guide in the $+x$ direction with wave vector, $A_{0}$ and entering a rough surface region of length, $L(0<x<1)$ where the rough boundaries are at $y=W+f_{+}(x)$ and $y=f-(x)$. The incident wave, $\psi_{\mathrm{m}}$, which interacts with roughness is scattered into other modes, $\psi_{\mathrm{sc}}$. The total field, $\psi$, is the surface of incident field and the scattered field

$$
\psi=\psi_{\text {in }}+\psi_{\text {sc }}
$$

Now to get an expression of $\psi_{\mathrm{sc}}$ and $\psi_{\text {in }}$ one has to calculate transmission coefficient for spheroidal dust particles.

\subsection{Scattering by spheroidal dust particles through moistured rough surface}

The expression for scattered field from a moisture rough surface for spheroidal dust particles obtained from the concept of radiation forms short current filament and thus the vector potential $A$ may be given as (Hollinger 1971),

$$
A=\frac{\mu_{0} \mathrm{Idle}^{-j} K_{0} r}{4 \pi r} a_{z}
$$

Now the value of $A$ in terms of the components in spheroidal polar coordinates can be written as

$$
A=\frac{\mu_{0}}{4 \pi r} \operatorname{Idle}^{-j} K_{0} r\left(a_{\mathrm{r}} \cos \theta-a_{0} \sin \theta\right),
$$

where

$$
a_{z}=a_{\mathrm{r}} \cos \theta-a_{0} \sin \theta .
$$

Further, using the relation

$$
E=-j \omega A+\frac{\nabla \nabla \cdot A}{j \omega \mu_{0} \varepsilon_{0}},
$$

then the value of scattered field

$$
\begin{aligned}
E & =-j \omega A \cdot \frac{\nabla \nabla \cdot A}{j \omega \mu_{0} \varepsilon_{0}} \\
& =\frac{j Z_{0} \mathrm{Idl}}{2 \pi K_{0}} \cos \theta\left(\frac{j K_{0}}{r^{2}}+\frac{1}{r^{3}}\right) e^{-j} K_{0} r a_{\mathrm{r}} \\
& -\frac{j Z_{0} \mathrm{Idl}}{4 \pi K_{0}} \sin \theta\left(\frac{-K^{2}}{r}+\frac{j K_{0}}{r^{2}}+\frac{1}{r^{3}}\right) e^{-j} K_{0} r a_{\theta} \\
& =E_{\mathrm{r}} a_{\mathrm{r}}+E_{\theta} a_{\theta},
\end{aligned}
$$

where $r$ is the larger relation to the wavelength, $\lambda_{0}$.

The only important terms are those that vary as $1 / r$, then far zone or radiated field may be given as

$$
E=j Z_{0} \mathrm{Idl} \sin \theta \frac{e^{-j} K_{0} r}{4 \pi r} a_{\theta},
$$

also

$$
E_{\mathrm{s}}=-\omega Z_{0} K_{0} P_{0} \sin \theta \frac{e^{-j} K_{0} r}{4 \pi r} a_{\theta} .
$$

Thus the value of $P_{0}$ for spheroidal dust particles can be obtained as (Wang et al 1980),

$$
P_{0}=\frac{4}{3} \pi a^{3} \psi_{\text {in }}=4 \pi a^{3} \frac{k-1}{k+2} \varepsilon_{0} E_{0} a_{z} .
$$

Thus the value of $\psi_{\text {in }}$ can be written from (19) as follows

$$
\psi_{\text {in }}=\frac{P_{0}}{\frac{4}{3} \pi a^{3}}=3\left(\frac{k-1}{k+2} \varepsilon_{0} E_{0} a_{z}\right) .
$$




\subsection{Extinction cross-section for spheroidal dust particle} (prolate and oblate)

The extinction cross-section $\left(\sigma_{\text {ext }}\right)$ for spheroidal dust particle is given as

$$
\psi_{\text {in }}=\frac{V_{\text {spheroidal }}}{4 \pi} \psi_{\text {sc }},
$$

where $V$ is the spheroidal volume of the spheroidal dust particle.

Now, for prolate spheroidal dust particle for volume, $V$, is given as

$$
V_{\text {spheroidal (oblate) }}=\frac{4}{3} \pi a^{2} a_{3},
$$

where

$$
\psi_{\mathrm{sc}}=\left(\ell_{z}+\frac{1}{\varepsilon-1}\right)^{-1},
$$

then the value $S(O, D)$ can be obtained as

$$
S(O, D)=K^{2}\left(\frac{V_{\text {spheroidal }}}{4 \pi} \psi_{\mathrm{sc}}\right)-j\left(K^{5}\right)\left(\frac{V_{\text {spheroidal }}}{4 \pi} \psi_{\mathrm{sc}}\right)^{2},
$$

further, combining (20) and (21), one can modify as follows

$$
\begin{gathered}
\sigma_{(\mathrm{ext})}=\frac{4 \pi}{R} R_{\mathrm{e}}\left[K^{2}\left(\frac{V_{\text {spheroidal }}}{4 \pi} \psi_{\mathrm{sc}}\right)-j\left(K^{5}\right)\left(\frac{V_{\text {spheroidal }}}{4 \pi} \psi_{\mathrm{sc}}\right)\right] \\
\sigma_{(\mathrm{ext})}=\frac{4 \pi}{K} R_{\mathrm{e}}\left[K ^ { 2 } \left(\frac{V_{\text {spheroidal }}}{4 \pi}\left(\ell_{z}-\frac{1}{\varepsilon-1}\right)^{-1}\right.\right. \\
\left.\left.-j\left(K^{5}\right) \frac{V_{\text {spheroidal }}}{4 \pi}\left(\ell_{z}-\frac{1}{\varepsilon-1}\right)\right)\right] .
\end{gathered}
$$

Solving (22), one has

$$
\begin{aligned}
\sigma_{(\text {ext })}= & \frac{4 \pi}{K}\left[\frac{K_{\text {spheroidal }}^{2}}{4 \pi} \frac{\varepsilon_{z} \ell_{z}}{\varepsilon_{1}^{2}+\varepsilon_{2}^{2}}\right. \\
& \left.-\frac{K^{5} V_{\text {spheroidal }}}{4 \pi}\left(2 \varepsilon_{1} \varepsilon_{2}+\ell_{z} \varepsilon_{2}\right)\right] .
\end{aligned}
$$

\section{Numerical computation}

Using (9), (20) and (24), the value of absorption loss, extinction cross-section and scattering field have been calculated and hence the data have been plotted in the form of graphs.

\section{Results and discussion}

We have investigated the cause of decrease in the scattered power for low frequency and thus resulting into low extinction scattering cross-section. (The scattering and extinction cross-section for the spheroidal (prolate and oblate) dependence on the incident elastic wave through rough surface with moisture percentages of 2 to $4 \cdot 5$.) The scattering mode reduces the frequencies on the percentage rough surface with parameters of moisture and size of the dust particles. We find that the effects of scattering is always significant for reducing the frequency below the spheroidal size over the particle size. Considering the simplicity of our model the results agree well with the theoretical considerations. The mode of dependence has

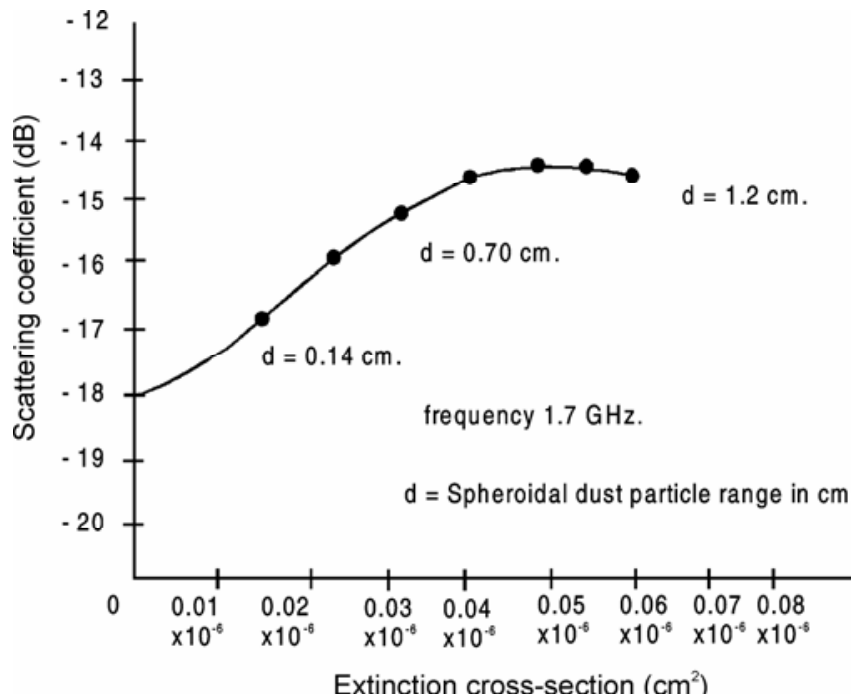

Figure 2. Response of back scattering coefficient and extinction cross-section for spheroidal dust particles in moisture rough surface at $1 \cdot 2 \mathrm{GHz}$.

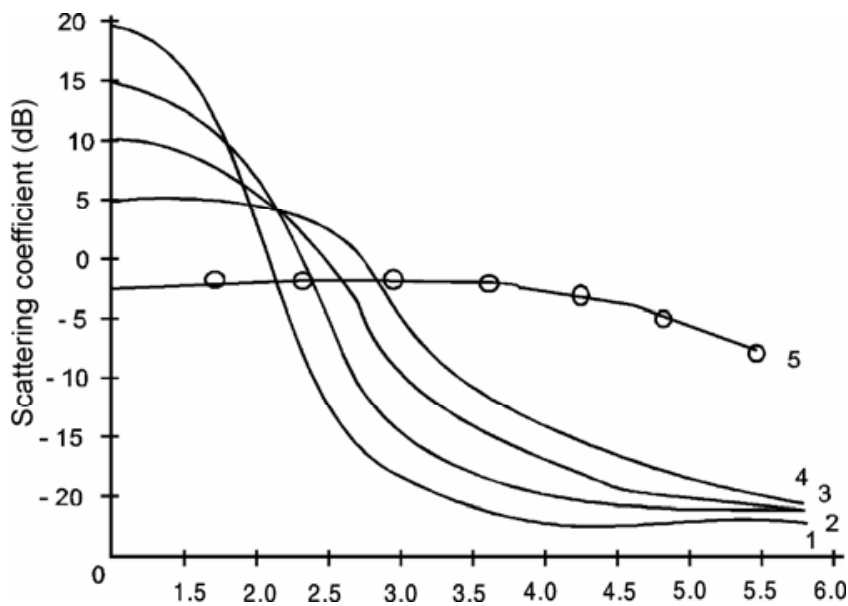

Figure 3. Pattern of scattering coefficient at $1 \mathrm{GHz}$ for spheroidal dust particles with different moisture rough surfaces. 
been plotted in the form of graphs in figures 2 and 3, respectively in the last section of this paper.

\section{References}

Backman P and Spizzichino 1987 The scattering of electromagnetic wave from rough surfaces (Nerwood, Massachusetts: Artech House)
Dobson M C and Ulaby F T 1981 IEEE Trans. Geosci.-Rem. Sens. GE-19 51

Fawwaz T, Laby U, Moore Richard K and Fung A K 1981 Radar remote sensing and surface scattering and emission theory (Addison Wesley Pub. Co.) Vol. II

Hollinger J P 1971 IEEE Trans. Geosci. Electrons GE-9 165

Shimaru J 1978 Wave propagation and scattering in random media (Academic Press) Chapters 2, 4, 6 and 14, Vols 1 \& 2

Wang J R, Shine J C and Mc Murtrey J E 1980 J. Phys. Res. 10801 